\title{
SYSTEMS OF NONLINEAR VOLTERRA EQUATIONS WITH POSITIVE DEFINITE KERNELS

\author{
BY
}

OLOF J. STAFFANS

\begin{abstract}
We study the boundedness and the asymptotic behavior of the solutions of a nonlinear, $\mathbf{R}^{n}$-valued Volterra equation with a positive definite kernel, generalizing earlier scalar results.
\end{abstract}

1. Introduction. The boundedness and the asymptotic behavior of the solutions of the nonlinear Volterra equation

$$
x^{\prime}(t)+\int_{[0, t]} g(x(t-s)) d \mu(s)=f(t) \quad\left(t \in \mathbf{R}^{+}\right) ; \quad x(0)=x_{0},
$$

has been studied in different contexts. Best known is naturally the classical, real case, i.e. when $x, g$ and $f$ are real functions, and $\mu$ is a real measure. Some scalar results have been generalized to equations in $\mathbf{R}^{n}$ (e.g. [6, §18] and [18]), and others have been proved directly for $\mathbf{R}^{n}$-valued equations (e.g. [5]), but on the whole the $\mathbf{R}^{n}$ theory is less developed than the scalar theory. Lately much effort has been devoted to the study of (1.1) in real Hilbert spaces (e.g. [1], [2], and [7]-[10]), and these results, of course, also apply to equations in $\mathbf{R}^{n}$. However, in an infinite-dimensional space certain additional difficulties show up which are not present in $\mathbf{R}^{n}$, and this may account for the fact that the Hilbert space results do not seem to be very sharp in $\mathbf{R}^{n}$, or at best unnecessarily complicated.

The preceding facts have motivated us to study (1.1) specifically in $\mathbf{R}^{n}$. We take $f \in L^{1}\left(\mathbf{R}^{+} ; \mathbf{R}^{n}\right), g \in C\left(\mathbf{R}^{n} ; \mathbf{R}^{n}\right)$, and let $\mu$ be a matrix-valued measure (think of $\mu$ as a matrix whose entries are scalar-valued measures). If $\mu$ is absolutely continuous, say $d \mu(t)=A(t) d t$, where $A$ is an i ntegrable, matrixvalued function, then one natural way to write (1.1) in $\mathbf{R}^{n}$ is

$$
x^{\prime}(t)+\int_{[0, t]} A(s) g(x(t-s)) d s=f(t) \quad\left(t \in \mathbf{R}^{+}\right) ; \quad x(0)=x_{0},
$$

where $x, g$ and $f$ have been identified with column vectors, and an ordinary matrix multiplication notion is used. In the general case we write (1.1) in the form

Received by the editors December 24, 1975.

AMS (MOS) subject classifications (1970). Primary 45D05, 45G99, 45M05. 
(E) $x^{\prime}(t)+\int_{[0, t]} d \mu(s) g(x(t-s))=f(t) \quad\left(t \in \mathbf{R}^{+}\right) ; \quad x(0)=x_{0}$, thereby understanding

$$
x_{j}^{\prime}(t)+\sum_{k=1}^{n} \int_{[0, t]} g_{k}(x(t-s)) d \mu_{j k}(s)=f_{j}(t) \quad(j=1, \ldots, n) .
$$

One very fruitful approach in the scalar case employs the notion of a positive definite kernel, and we use the same approach in $\mathbf{R}^{\boldsymbol{n}}$. Many of the scalar results go through without any difficulties whatsoever, and others only require a small amount of additional work. However, certain new problems arise in $\mathbf{R}^{n}$ which are not present in $\mathbf{R}^{\mathbf{1}}$, and we try to pay particular attention to these.

We begin in $\$ 3$ by characterizing the positive definite kernels in $\mathbf{R}^{\boldsymbol{n}}$. No particular difficulties show up, but one interesting question presents itself: To which extent is a positive definite kernel selfadjoint? We show that the a priori symmetry requirements on the kernel which are widely used in papers on equations in Hilbert spaces are superfluous (we only discuss $\mathbf{R}^{n}$, but the same method works in a Hilbert space). However, the mere fact that a kernel is positive definite implies a certain amount of selfadjointness (Remark 3.3 below).

Another interesting observation is that one aspect of the theory is simpler in $\mathbf{C}^{n}$ than in $\mathbf{R}^{n}$ (Remark 3.2 below).

In $\S 4$ we investigate what the boundedness of a particular quadratic integral implies about the asymptotic behavior of the integrated function. As in the scalar case, this is a key step in the development of a theory on the asymptotic behavior of the bounded solutions of (E). One who is familiar with the scalar theory presented in [15] finds no surprises in $\$ 4$.

We get to the heart of the matter in $\$ 5$, where we begin to apply the results of $\$ \S 3-4$ to the study of $(E)$. We give one theorem on existence and boundedness of solutions of (E), and two theorems on the asymptotic spectra of the bounded solutions of (E). Here we face a well-known difficulty: In order for the proofs in the scalar case to carry over to $\mathbf{R}^{n}$, we have to suppose that $g$ is the gradient of a function $G$ (which is trivially true in $R^{1}$ ). This assumption must not be regarded as a technical one; it is indispensable, as we show with a linear counterexample.

Theorem 3.2 in [16], which gives sufficient conditions for the solutions of the scalar equation (1.1) to satisfy $g(x(t)) \rightarrow 0(t \rightarrow \infty)$, also generalizes to $\mathbf{R}^{n}$, as we show in $\S 6$. The most natural way of generalizing [16, Theorem 3.2] does not lead to a satisfactory result, since it does not contain the case when $\mu$ is the derivative of a decreasing matrix function (see the example following Theorem 6.1 below). A less obvious extension (Theorem 6.2 below) turns out to be substantially better. 
Finally, in $\$ 7$ we show that two different types of monotone kernels are positive definite. Also this is analogous to the corresponding scalar result. In addition we investigate the annoying case when the kernel of $(E)$ is degenerate and some of our theorems either become trivial or do not apply. We conclude by presenting the result one gets when one applies, e.g., Theorem 6.1 to a first order, nonlinear system of ordinary differential equations.

2. Preliminaries. Most of the notations we use are so standardized in the mathematical literature that we do not feel a need to explain them one by one. However, we also employ some less common notations, and these are explained below.

The following symbols are given a special meaning:

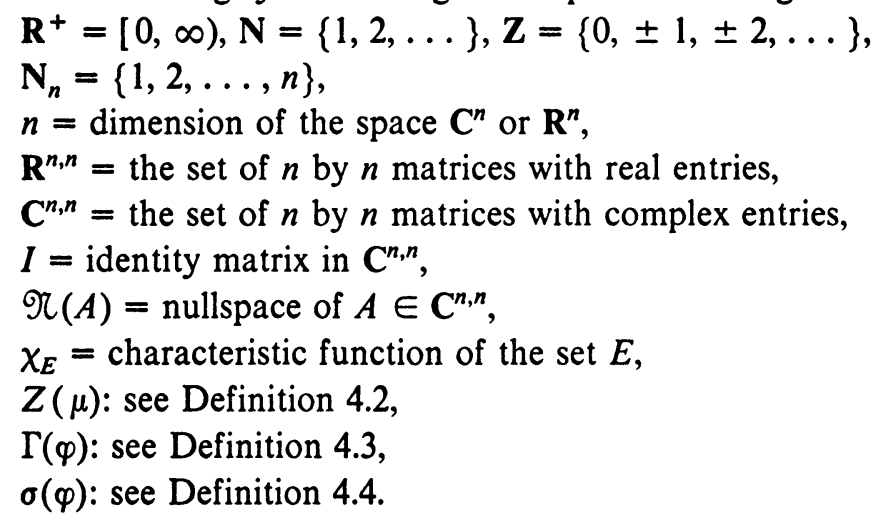

Whenever $F$ denotes a function space, we mean by $F(X ; Y)$ those functions that map $X$ into $Y$. A similar notation is used for measure and distribution spaces. Examples on such spaces are:

BUC: Bounded, uniformly continuous functions,

LAC: Locally absolutely continuous functions,

BM: (Finite) Borel measures,

M: Radon measures (locally BM),

$\delta$ : Rapidly decreasing functions,

$\delta$ ': Tempered distributions,

PD: See Definitions 3.1 and 3.2.

In particular, we work with $\mathbf{C}^{n}$ - and $\mathbf{C}^{n, n}$-valued measures and distributions. The simplest way to visualize a $\mathbf{C}^{n}$ - or $\mathbf{C}^{n, n}$-valued distribution is to think of a vector or matrix whose entries are scalar-valued distributions, and perform all calculations componentwise.

The operator ${ }^{*}$ operates on elements in $\mathbf{C}^{n, n}$ in a standard way: $A^{*}$ is the adjoint of $A \in \mathrm{C}^{n, n}$. We identify $\mathbf{C}^{n}$ with the set of $n$ by 1 matrices, and let ${ }^{*}$ operate on elements of $\mathbf{C}^{n}$ as well: $\alpha^{*}$ is the complex conjugate of the transpose of $\alpha$. This notation is also carried over to $\mathbf{C}^{n}$ - and $\mathbf{C}^{n, n}$-valued functions and distributions. 
Subindexes are mainly used to denote the components of an element in $\mathbf{C}^{n}$ or $\mathbf{C}^{n, n}$, but occasionally for other purposes when no confusion is likely to arise. Scalar-, vector- and matrix-valued functions and distributions are multiplied following the standard rules for matrix multiplication. In particular, the integral

$$
\int_{[0, T]} \varphi^{*}(t) \int_{[0, t]} d \mu(s) \varphi(t-s) d t
$$

should be interpreted as

$$
\sum_{j, k \in N_{n}} \int_{[0, T]} \bar{\varphi}_{j}(t) \int_{[0, t]} \varphi_{k}(t-s) d \mu_{j k}(s) d t .
$$

In addition to the matrix operator * we need some operators which operate specifically on functions or measures (the integrals are over $\mathbf{R}$ or $\mathbf{R}^{+}$, and $d t$ throughout stands for Lebesgue measure):

' (prime): differentiation,

$\tau_{h}$ (translation): $\left(\tau_{h} \varphi\right)(t)=\varphi(t+h)$,

- (composition): $(g \circ \varphi)(t)=g(\varphi(t))$,

* (convolution):

$$
\begin{array}{ll}
(\varphi * \lambda)(t)=\int \varphi(t-s) d \lambda(s) & (\varphi \in \mathrm{BUC}, \lambda \in \mathrm{BM}), \\
(\varphi * h)(t)=\int \varphi(t-s) h(s) d s & \left(\varphi \in L^{\infty}, h \in L^{1}\right)
\end{array}
$$

- (Fourier transform):

$$
\begin{array}{ll}
\hat{\lambda}(\omega)=\int e^{-i \omega s} d \lambda(s) & (\lambda \in \mathrm{BM}), \\
\hat{h}(\omega)=\int e^{-i \omega s} h(s) d s & \left(h \in L^{1}\right),
\end{array}
$$

$\because \tilde{\varphi}=(\varphi \circ \eta)^{*}$, where $\eta(t)=-t$ maps $\mathbf{R} \rightarrow \mathbf{R}$.

Note in particular the definition of $\sim$; it is one possible extension (and the right one for our purposes) of the scalar operator used in [14]. We allow the same operators to operate on distributions, thereby understanding that they have been extended in the standard way.

The Euclidean norm in $\mathbf{C}^{n}$ is denoted by || , and the corresponding operator norm in $\mathbf{C}^{n, n}$ by \|\| . $\mathbf{R}^{+}$.

Functions and measures defined on $\mathbf{R}^{+}$are extended to $\mathbf{R}$ by zero outside

The words "positive", "negative", "increasing" and "decreasing" are used as synonyms to "nonnegative", "nonpositive", "nondecreasing" and "nonincreasing".

By a positive matrix $A(A>0)$ we mean one which satisfies $\alpha^{*} A \alpha>0$ $\left(\alpha \in \mathbf{C}^{n}\right)$, i.e. a matrix which sometimes is called positive semidefinite. In 
particular, since we allow $\alpha$ to take values in $\mathbf{C}^{n}$, every positive matrix is selfadjoint. We frequently use the well-known fact that if $A>0, \alpha \in \mathrm{C}^{n}$, then $\alpha^{*} A \alpha=0$ iff $A \alpha=0$.

The same positivity concept carries over to $\mathbf{C}^{n, n}$-valued functions and measures. The positivity of a $\mathbf{C}^{n, n}$-valued function is tested pointwise, and we call a $\mathbf{C}^{n, n}$-valued measure $\mu$ positive $(\mu \geqslant 0)$ if $\mu(E) \geqslant 0$ for every bounded Borel set $E$.

3. Positive definite, matrix-valued measures. Our first goal is to extend the results in $[14, \S 1]$ to matrix-valued measures. The first choice which faces us is whether one should work in $\mathbf{R}^{n}$ or in $\mathbf{C}^{n}$. The natural setting in the applications to Volterra equations is $\mathbf{R}^{n}$. On the other hand, we want to take Fourier transforms, and therefore we need complex scalars. Moreover, the theory of positive definiteness is no more complicated in $\mathbf{C}^{n}$ than in $\mathbf{R}^{n}$, on the contrary, it is simpler (cf. Remark 3.2 below). For these reasons we basically work in $\mathbf{C}^{n}$.

Definition 1.2 in [14] generalizes trivially to $\mathbf{C}^{n}$ :

Definition 3.1. A measure $\mu \in \mathbf{M}\left(\mathbf{R}^{+} ; \mathbf{C}^{n, n}\right)$ is positive definite $(\mu \in$ $\left.\operatorname{PD}\left(\mathbf{R}^{+} ; \mathbf{C}^{n, n}\right)\right)$ if for every $T \in \mathbf{R}^{+}$and for every $\varphi \in C\left([0, T] ; \mathbf{C}^{n}\right)$,

$$
\operatorname{Re}\left\{\int_{[0, T]} \varphi^{*}(t) \int_{[0, t]} d \mu(s) \varphi(t-s) d t\right\} \geqslant 0 .
$$

One defines $\operatorname{PD}\left(\mathbf{R}^{+} ; \mathbf{R}^{n, n}\right)$ as above, replacing $\mathbf{C}^{n, n}$ by $\mathbf{R}^{n, n}$ and $\mathbf{C}^{n}$ by $\mathbf{R}^{n}$. It is easy to see that $\operatorname{PD}\left(\mathbf{R}^{+} ; \mathbf{R}^{n, n}\right)=\mathrm{M}\left(\mathbf{R}^{+} ; \mathbf{R}^{n, n}\right) \cap \operatorname{PD}\left(\mathbf{R}^{+} ; \mathbf{C}^{n, n}\right)$.

As a reader familiar with, e.g., [14] might expect, the positive definiteness of $\mu$ is related to the behavior of $\hat{\mu}$. Some results in this direction are known even in the infinite-dimensional case (i.e. with $\mathbf{C}^{n}$ replaced by a Hilbert space), e.g., MacCamy's and Wong's [10, Lemma (4.3)] applies when $d \mu(t)=$ $A(t) d t\left(t \in \mathbf{R}^{+}\right)$, where $A \in C^{2}\left(\mathbf{R}^{+} ; \mathbf{R}^{n, n}\right), A-A(\infty), A^{\prime}, A^{\prime \prime} \in L^{1}$, and $A=A^{*}$. This lemma (applied to the finite dimensional case) states that under the preceding assumptions, $\mu$ is positive definite if $A(\infty) \geqslant 0$, and

$$
\operatorname{Re}\left\{(A-A(\infty))^{\wedge}\right\} \geqslant 0 .
$$

The one completely new condition which MacCamy and Wong use, compared to the $\mathbf{R}^{1}$ case, is that $\mu$ be selfadjoint. The same condition is found throughout in papers on Hilbert space theory. When one wants to extend results in $\mathbf{R}^{1}$ to $\mathbf{R}^{n}$ it is indeed tempting to assume selfadjointness, as this makes the proofs for the $\mathbf{R}^{1}$ case go through without difficulties in $\mathbf{R}^{n}$. However, the theory in [14] is a theory for $\mathbf{C}^{1}$, not $\mathbf{R}^{1}$, and no "selfadjointness", i.e. real-valuedness, is assumed of $\mu$. Following the procedure in [14] one gets a natural extension to $\mathbf{C}^{n}$ : 
Definition 3.2. A measure $\nu \in \mathrm{M}\left(\mathbf{R} ; \mathbf{C}^{n, n}\right)$ is positive definite ( $\nu \in$ $\left.\mathrm{PD}\left(\mathbf{R} ; \mathbf{C}^{n, n}\right)\right)$ if for every $T \in \mathbf{R}^{+}$and for every $\varphi \in L^{2}\left([-T, T] ; \mathbf{C}^{n}\right)$,

$$
\int_{[-T, T]} \varphi^{*}(t) \int_{[t-T, t+T]} d \nu(s) \varphi(t-s) d t \geqslant 0 .
$$

THEOREM 3.1. Let $\mu \in \mathrm{M}\left(\mathbf{R}^{+} ; \mathbf{C}^{n, n}\right)$, and define $\nu=\mu+\tilde{\mu}$. Then $\mu \in$ $\operatorname{PD}\left(\mathbf{R}^{+} ; \mathbf{C}^{n, n}\right)$ iff $\nu \in \operatorname{PD}\left(\mathbf{R} ; \mathbf{C}^{n, n}\right)$.

The proof of Theorem 3.1 is the same as (or rather somewhat simpler than) the proof of [14, Theorem 1.1]. In particular, it uses the fact that

$$
\begin{aligned}
\operatorname{Re}\left\{\int_{[0, T]} \varphi^{*}(t) \int_{[0, t]} d \mu(s) \varphi(t-s) d t\right\} \\
=\frac{1}{2} \int_{[0, T]} \varphi^{*}(t) \int_{[t-T, t]} d \nu(s) \varphi(t-s) d t
\end{aligned}
$$

for every $T \in \mathbf{R}^{+}$, and for every $\varphi \in C\left([0, T] ; \mathbf{C}^{n}\right)$.

REMARK 3.1. As in [14] we observe that the mapping $\mu \rightarrow \nu$ given by $\nu=\mu+\tilde{\mu}$ is not one-to-one. The value of $\mu(\{0\})-\mu^{*}(\{0\})$ is lost, and $\nu(\{0\})=\mu(\{0\})+\mu^{*}(\{0\})$ is selfadjoint. This fact enables us to apply the theory of positive definiteness to a first order system of differential equations with no symmetry requirement on the right-hand side. See Proposition 7.4 below.

We have the following characterization of positive definiteness:

TheOREM 3.2. Let $\nu \in M\left(R ; \mathbf{C}^{n, n}\right)$. Then the following three statements are equivalent:

(i) $\nu \in \mathrm{PD}\left(\mathbf{R} ; \mathbf{C}^{n, n}\right)$,

(ii) $\alpha^{*} \nu \alpha \in \mathrm{PD}(\mathbf{R} ; \mathbf{C})$ for every $\alpha \in \mathbf{C}^{n}$,

(iii) $\nu \in \mathcal{S}^{\prime}\left(\mathbf{R} ; \mathbf{C}^{n, n}\right), \hat{\nu} \in \mathrm{M}\left(\mathbf{R} ; \mathbf{C}^{n, n}\right)$, and $\hat{\nu} \geqslant 0$.

The proof of Theorem 3.2 is deferred to the end of this section.

Combining Theorems 3.1 and 3.2 with the fact that $\nu=\mu+\tilde{\mu}$ implies $\hat{\nu}=\hat{\mu}+\hat{\mu}^{*}$ (do the computation componentwise, and see [12, p. 251]) one obviously gets

Corollary 3.1. Let $\mu \in \mathrm{M}\left(\mathbf{R}^{+} ; \mathbf{C}^{n, n}\right)$. Then the following three statements are equivalent:

(i) $\mu \in \mathrm{PD}\left(\mathbf{R}^{+} ; \mathbf{C}^{n, n}\right)$,

(ii) $\alpha^{*} \mu \alpha \in \mathrm{PD}\left(\mathbf{R}^{+} ; \mathbf{C}\right)$ for every $\alpha \in \mathbf{C}^{n}$,

(iii) $\mu \in \mathcal{S}^{\prime}\left(\mathbf{R} ; \mathbf{C}^{n, n}\right), \hat{\mu}+\hat{\mu}^{*} \in \mathbf{M}\left(\mathbf{R} ; \mathbf{C}^{n, n}\right)$, and $\hat{\mu}+\hat{\mu}^{*} \geqslant 0$.

Whenever $\mu$ is selfadjoint and $\mathbf{R}^{n, n}$-valued, then $\operatorname{Re} \hat{\mu}=\frac{1}{2}\left(\hat{\mu}+\hat{\mu}^{*}\right)$. Thus Corollary 3.1 contains the $\mathbf{R}^{n}$ version of [10, Lemma (4.3)] $(A(\infty) \neq 0$ only means that $\operatorname{Re} \hat{\mu}$ has a positive "point mass" at zero; cf. [16, Lemma 1.1]). 
Corollary 3.1 also contains the $\mathbf{R}^{\boldsymbol{n}}$ version of [9, Theorem 2.1]. The relation between $A$ in [9, Theorem 2.1] and $\mu$ in Corollary 3.1 is $A(t)=\mu([0, t])$ $\left(t \in \mathbf{R}^{+}\right)$.

REMARK 3.2. Corollary 3.1 illustrates how the theory of positive definiteness is simpler in $\mathbf{C}^{n}$ than in $\mathbf{R}^{n}$ : The version of Corollary 3.1 one gets by replacing $\mathbf{C}^{n}, \mathbf{C}^{n, n}$ by $\mathbf{R}^{n}, \mathbf{R}^{n, n}$ is false. Take, e.g., $n=2, d \mu(t)=A d t\left(t \in \mathbf{R}^{+}\right)$, where $A=\left(\begin{array}{c}0 \\ 1\end{array}-1\right)$. Then $\alpha^{*} A \alpha=0\left(\alpha \in \mathbf{R}^{2}\right)$, so in particular $\alpha^{*} \mu \alpha \in \operatorname{PD}\left(\mathbf{R}^{+} ; \mathbf{R}\right)$ $\left(\alpha \in \mathbf{R}^{2}\right)$. However, $\mu$ is not positive definite. Take $T=2 \pi, \varphi^{*}(t)=$ $(\sin (t), \cos (t))$, and compute

$$
\int_{[0, T]} \varphi^{*}(t) \int_{[0, t]} A \varphi(t-s) d s d t=-2 \pi<0 .
$$

REMARK 3.3. There is an even simpler way to see that the example in Remark 3.2 is not positive definite. It suffices to observe that $A$ is not selfadjoint. Although we have not a priori assumed selfadjointness of a positive definite measure $\mu$, the mere fact that $\mu$ is positive definite implies a certain amount of selfadjointness. Take, e.g., the case when $d \mu(t)=A(t) d t$ $\left(t \in \mathbf{R}^{+}\right)$, where $A \in C\left(\mathbf{R}^{+} ; \mathbf{C}^{n, n}\right)$. Then $A(0) \geqslant 0$, hence $A(0)$ is selfadjoint. In view of Corollary 3.1 the proof of this fact can be reduced to the corresponding scalar statement that every $a \in C\left(\mathbf{R}^{+} ; \mathbf{C}\right) \cap \operatorname{PD}\left(\mathbf{R}^{+} ; \mathbf{C}\right)$ satisfies $a(0) \geqslant 0$. This scalar version is true because every bounded function $b \in \mathrm{PD}(\mathbf{R} ; \mathbf{C})$ is a.e. equal to a continuous function (the extended $b$ defined by $b(t)=a(t)\left(t \in \mathbf{R}^{+}\right), b(t)=\bar{a}(-t)(t<0)$ could a priori have a discontinuity at zero), and every $b \in C(\mathbf{R} ; \mathbf{C}) \cap \mathrm{PD}(\mathbf{R} ; \mathbf{C})$ (positive definite in the sense of Bochner) satisfies $b(0) \geqslant 0$. A similar observation to the preceding one is the following: If $\mu$ is of the form $d \mu(t)=d \lambda(t)+A d t\left(t \in \mathbf{R}^{+}\right)$, where $\lambda \in \mathrm{BM}\left(\mathbf{R}^{+} ; \mathbf{C}^{n, n}\right)$ and $A \in \mathbf{C}^{n, n}$, then $\mu$ is positive definite iff $\lambda$ is positive definite and $A \geqslant 0$ (hence $A=A^{*}$ ). Compute $\hat{\mu}=\hat{\lambda}+A\left[\pi \delta_{0}-i h\right]$, where $\delta_{0}$ is the Dirac measure at zero, and $h$ is the Hilbert transform. Thus $\hat{\mu}+\hat{\mu}^{*}$ has order zero iff $A$ is selfadjoint, and the point mass at zero is positive iff $A>0$.

Proof of Theorem 3.2. That (i) $\Rightarrow$ (ii) is trivial.

Suppose that (ii) holds. We want to show that (iii) then is true. By (ii), all diagonal elements $\nu_{j j}$ of $\nu$ are scalar-valued, positive definite measures, and so by $\left[14\right.$, Theorem 1.2], $\nu_{i j} \in \mathcal{S}^{\prime}(\mathbf{R} ; \mathbf{C}), \hat{\nu}_{j j} \in \mathbf{M}(\mathbf{R} ; \mathbf{C})\left(j \in \mathbf{N}_{n}\right)$. That also every nondiagonal element $\nu_{j k}$ satisfies $\nu_{j k} \in \mathcal{S}^{\prime}(\mathbf{R} ; \mathbf{C}), \hat{\nu}_{j k} \in \mathrm{M}(\mathbf{R} ; \mathbf{C})$ follows from the fact that for every $\alpha, \beta \in \mathbf{C}^{n}$,

$$
\begin{aligned}
4 \alpha^{*} \nu \beta= & (\alpha+\beta)^{*} \nu(\alpha+\beta)-(\alpha-\beta)^{*} \nu(\alpha-\beta) \\
& -i(\alpha+i \beta)^{*} \nu(\alpha+i \beta)+i(\alpha-i \beta)^{*} \nu(\alpha-i \beta),
\end{aligned}
$$

and here the right-hand side is a combination of positive definite measures. Thus $\nu \in \mathcal{S}^{\prime}\left(\mathbf{R} ; \mathbf{C}^{n, n}\right)$ and $\hat{\nu} \in \mathrm{M}\left(\mathbf{R} ; \mathbf{C}^{n, n}\right)$. 
It still remains to show that $\hat{\nu}(E) \geqslant 0$, i.e. $\alpha^{*} \hat{\nu}(E) \alpha \geqslant 0\left(\alpha \in \mathbf{C}^{n}\right)$, for every bounded Borel set $E$. However, this also follows from (ii) combined with [14, Theorem 1.2], because the scalar-valued measure $\alpha^{*} \hat{\nu} \alpha$ is positive for every $\alpha \in \mathbf{C}^{n}$.

To prove that (iii) $\Rightarrow$ (i) one first uses an approximation argument to show that $\int_{\mathbf{R}} \eta^{*}(\omega) d \hat{\nu}(\omega) \eta(\omega) \geqslant 0$ for every $\eta \in C\left(\mathbf{R} ; \mathbf{C}^{n}\right)$ with compact support (such functions can be uniformly approximated by step functions), and then one completes the proof as in the scalar case.

4. An asymptotic problem. We continue as in [15] by studying the asymptotic problem: What does the condition

$$
\sup _{T \in \mathbf{R}^{+}} \operatorname{Re}\left\{\int_{[0, T]} \varphi^{*}(t) \int_{[0, t]} d \mu(s) \varphi(t-s) d t\right\}<\infty
$$

imply about the asymptotic behavior of $\varphi$ ? No unexpected difficulties show up.

Definition 4.1. A measure $\lambda \in \mathrm{M}\left(\mathbf{R} ; \mathbf{C}^{n, n}\right)$ is strictly positive at a point $\omega \in \mathbf{R}$ if there exists $\varepsilon>0$ such that the measure $\lambda_{\varepsilon}$ defined by $d \lambda_{\varepsilon}(t)=d \lambda(t)$ $-\varepsilon I d t$ is positive in $(\omega-\varepsilon, \omega+\varepsilon)$.

DEFINITION 4.2. The spectral set $Z(\mu)$ of a measure $\mu \in \mathrm{PD}\left(\mathbf{R}^{+} ; \mathbf{C}^{n, n}\right)$ is the set where $\hat{\mu}+\hat{\mu}^{*}$ is not strictly positive.

Definition 4.3. The limit set $\Gamma(\varphi)$ of a function $\varphi \in L^{\infty}\left(\mathbf{R}^{+} ; \mathbf{C}^{n}\right)$ is given by

$$
\begin{aligned}
\Gamma(\varphi)=\left\{\psi \in L^{\infty}\left(\mathbf{R} ; \mathbf{C}^{n}\right) \mid \tau_{t_{k}} \varphi \rightarrow\right. & \psi \text { weak }{ }^{*} \text { in } L^{\infty}\left(\mathbf{R} ; \mathbf{C}^{n}\right), \\
& \text { for some sequence } \left.t_{k} \rightarrow \infty\right\} .
\end{aligned}
$$

As in the scalar case (see $[15, \S 2])$ one can show that whenever $\varphi \in$ $\operatorname{BUC}\left(\mathbf{R}^{+} ; \mathbf{C}^{n}\right)$, then $\Gamma(\varphi)$ is also given by

$$
\begin{array}{r}
\Gamma(\varphi)=\left\{\psi \in \mathrm{BUC}\left(\mathbf{R} ; \mathbf{C}^{n}\right) \mid \tau_{t_{k}} \varphi \rightarrow \psi\right. \text { uniformly on compact sets, } \\
\text { for some sequence } \left.t_{k} \rightarrow \infty\right\} .
\end{array}
$$

Definition 4.4. The spectrum $\sigma(\varphi)$ of a function $\varphi \in L^{\infty}\left(\mathbf{R} ; \mathbf{C}^{n}\right)$ is the support of the distribution Fourier transform $\hat{\varphi}$.

Note that if $\varphi_{j}\left(j \in \mathbf{N}_{n}\right)$ are the components of $\varphi$ in Definition 4.4 , then $\sigma(\varphi)=\cup_{j \in N_{n}} \sigma\left(\varphi_{j}\right)$, where $\sigma\left(\varphi_{j}\right)$ is the spectrum of the scalar function $\varphi_{j}$.

THEOREM 4.1. Let $\mu \in \operatorname{PD}\left(\mathbf{R}^{+} ; \mathbf{C}^{n, n}\right), \varphi \in L^{\infty}\left(\mathbf{R}^{+} ; \mathbf{C}^{n}\right)$, and suppose that (4.1) holds. Then $\sigma(\psi) \in Z(\mu)$ for every $\psi \in \Gamma(x)$.

Proof of Theorem 4.1. Take some $\omega \notin Z(\mu)$. Choose an $\varepsilon$ as in Definition 4.1 (with $\lambda=\hat{\mu}+\hat{\mu}^{*}$ ), and choose some $\eta \in \Omega(\mathbf{R} ; \mathbf{C})$ such that $0 \leqslant \hat{\eta} \leqslant$ $\varepsilon, \hat{\eta}(\omega)=\varepsilon, \sigma(\eta) \subset[\omega-\varepsilon, \omega+\varepsilon]$. Define $d \xi(t)=I \eta(t) d t\left(t \in \mathbf{R}^{+}\right)$. Then $\hat{\xi}+\hat{\xi}^{*}=I \hat{\eta}$, and hence by Corollary $3.1, \mu-\xi$ is positive definite. This, together with (4.1), gives 


$$
\sup _{T \in \mathbb{R}^{+}} \int_{[0, T]} \varphi^{*}(t) \int_{[0, t]} d \xi(s) \varphi(t-s) d t<\infty .
$$

Substituting $d \xi(s)=\operatorname{I} \eta(s) d s$, and using the fact that $\eta$ is positive definite, we get

$$
\sup _{\boldsymbol{T} \in \mathbf{R}^{+}} \int_{[0, T]} \bar{\varphi}_{j} \int_{[0, t]} \eta(s) \varphi_{j}(t-s) d s d t<\infty \quad\left(j \in \mathbf{N}_{n}\right) .
$$

Now take some $\psi \in \Gamma(\varphi)$. Note that $\psi_{j} \in \Gamma\left(\varphi_{j}\right)\left(j \in \mathbf{N}_{n}\right)$. Theorem 3.1 in [15] (together with [14, Theorem 1.1 and Lemma 1.1]) yields $\omega \notin \sigma\left(\psi_{j}\right)$ $\left(j \in \mathbf{N}_{n}\right.$ ), and thus $\omega \notin \sigma(\psi)$. This completes the proof of Theorem 4.1.

Similar proofs could obviously be used to extend [15, Theorems 4.1 and 4.3].

5. On the equation (E). We next apply the theory of $\$ \S 3-4$ to the nonlinear, vector-valued Volterra equation (E). Here we work in $\mathbf{R}^{n}$ and not in $\mathbf{C}^{n}$. A theory for $\mathbf{C}^{n}$ is obtained if one imbeds $\mathbf{C}^{n}$ in $\mathbf{R}^{2 n}$.

Our assumptions are the following:

$$
\begin{aligned}
& \qquad \mu \in \operatorname{PD}\left(\mathbf{R}^{+} ; \mathbf{R}^{n, n}\right), \\
& g \text { is the gradient of a function } G \in C^{1}\left(\mathbf{R}^{n} ; \mathbf{R}\right), \\
& \inf _{\xi \in \mathbf{R}^{n}} G(\xi)>-\infty, \sup _{\xi \in \mathbf{R}^{n}}|g(\xi)|(1+|G(\xi)|)^{-1}<\infty, \\
& f \in L^{1}\left(\mathbf{R}^{+} ; \mathbf{R}^{n}\right) .
\end{aligned}
$$

THEOREM 5.1. Let (H1)-(H4) hold. Then (E) has at least one solution $x$ on $\mathbf{R}^{+}$, and for each solution $x$ the function $g \circ x$ is bounded. If also $\lim _{|\xi| \rightarrow \infty} G(\xi)$ $=\infty$, then all solutions of $(\mathrm{E})$ are bounded.

The proof of Theorem 5.1 is the same as in the scalar case [11, §2] (line (2.4) in [11] becomes trivial because of ( $\mathrm{H} 1)$ ).

In the sequel we suppose that we have a bounded solution:

$$
x \in L^{\infty}\left(\mathbf{R}^{+} ; \mathbf{R}^{n}\right) \cap \operatorname{LAC}\left(\mathbf{R}^{+} ; \mathbf{R}^{n}\right) \text { satisfies (E) a.e. on } \mathbf{R}^{+} \text {. }
$$

THEOREM 5.2. Let (H1), (H2), (H4) and (H5) hold. Then $\sigma(\psi) \in Z(\mu)$ for every $\psi \in \Gamma(g \circ x)$.

The proof of Theorem 5.2 is also the same as in the scalar case [15, Proposition 7.1] (the part referring to [15, Theorem 3.1]).

We continue as in [16], and refine Theorem 5.2 in the case when $\mu$ has a finite total variation (for simplicity we take $\beta=0$ in [16, Condition (H1)]). We replace $(\mathrm{Hl})$ by

$$
\mu \in \operatorname{BM}\left(\mathbf{R}^{+} ; \mathbf{R}^{n, n}\right), \quad \hat{\mu}+\hat{\mu}^{*} \geqslant 0 .
$$


Note that by Corollary 3.1, (H6) implies (H1). When (H6) holds, then $\hat{\mu}$ is continuous, and therefore $Z(\mu)$ is also given by

$$
Z(\mu)=\left\{\omega \in \mathbf{R} \mid \hat{\mu}(\omega)+\hat{\mu}^{*}(\omega) \text { is singular }\right\} .
$$

If, in addition, (H2), (H4) and (H5) hold, then $x, g \circ x \in \mathrm{BUC}\left(\mathbf{R}^{+} ; \mathbf{R}^{n}\right)$, and one can use (4.2) to define $\Gamma(x), \Gamma(g \circ x)$. Moreover, every $y \in \Gamma(x)$ satisfies the limit equation

$$
y^{\prime}(t)+\int_{\mathbf{R}^{+}} d \mu(s) g(y(t-s))=0 \quad(t \in \mathbf{R}) .
$$

The proofs of these claims are the same as in the scalar case.

THEOREM 5.3. Let (H2), (H4), (H5) and (H6) hold. Then every $y \in \Gamma(x)$ satisfies $\sigma(y) \subset \sigma(g \circ y) \cup\{0\}$, and $\sigma(g \circ y) \subset Z(\mu)$.

The proof of Theorem 5.3 is a straightforward modification of the proof of [16, Theorem 3.1].

The set $\Gamma(x)$ has, in fact, more structure than what is evident from Theorem 5.3. This is completely analogous to the linear case discussed in [6, pp. 552-553]. Loosely speaking, for each $y \in \Gamma(x)$ the value of $(g \circ y)^{\wedge}$ at an isolated point $\omega$ of $Z(\mu)$ is contained in $\mathcal{\vartheta}\left(\hat{\mu}(\omega)+\hat{\mu}^{*}(\omega)\right)$. This observation (in a modified form) plays a key role in the proof of Theorem 6.2 below.

Taking $n=1$ in Theorems 5.1-5.3 the hypotheses essentially reduce to the customary scalar hypotheses. In particular, (H2) holds whenever $g \in$ $C(\mathbf{R} ; \mathbf{R})$; one can define $G(\xi)=\int_{0}^{\xi} g(\eta) d \eta(\xi \in \mathbf{R})$. As soon as $n \geqslant 2$ the condition $g \in C\left(\mathbf{R}^{n} ; \mathbf{R}^{n}\right)$ is strictly weaker than (H2). One can, of course, ask whether Theorems 5.2-5.3 remain true with (H2) replaced by $g \in C\left(\mathbf{R}^{n} ; \mathbf{R}^{n}\right)$ (Theorem 5.1 cannot even be formulated without $(\mathrm{H} 2)$ ). The answer is negative, as the following linear counterexample shows. Take $n=2, d \mu(t)=$ $I e^{-t} d t\left(t \in \mathbf{R}^{+}\right), g(\xi)=\left(\begin{array}{cc}1 & -1 \\ 1\end{array}\right) \xi$, and consider (E) with $f=0, x_{0}^{*}=(2,1)$. Clearly the kernel is positive definite, and $Z(\mu)=\varnothing$. The condition (H2) does not hold (for linear functions (H2) is equivalent to selfadjointness; then $G(\xi)=\frac{1}{2} \xi^{*} g(\xi)$ ). Interpret $\mathbf{R}^{2}$ as the complex plane, and define $z(t)=x_{1}(t)$ $+i x_{2}(t)\left(t \in \mathbf{R}^{+}\right)$. Then $z$ satisfies

$$
z^{\prime}(t)+(1+i) \int_{[0, t]} e^{-(t-s)} z(s) d s=0 \quad\left(t \in \mathbf{R}^{+}\right) ; \quad z(0)=2+i .
$$

This equation is equivalent to the second order differential equation

$$
z^{\prime \prime}+z^{\prime}+(1+i) z=0 ; \quad z(0)=2+i ; \quad z^{\prime}(0)=0,
$$

whose solution is

$$
z(t)=(1+i) e^{-i t}+e^{(i-1) t} \quad\left(t \in \mathbf{R}^{+}\right) .
$$

Returning to the original interpretation of $\mathbf{C}$ as $\mathbf{R}^{\mathbf{2}}$ we obtain 


$$
\begin{aligned}
x(t) & =\left[\begin{array}{l}
\left(1+e^{-t}\right) \cos (t)+\sin (t) \\
\cos (t)-\left(1-e^{-t}\right) \sin (t)
\end{array}\right] \quad\left(t \in \mathbf{R}^{+}\right), \\
g(x(t)) & =\left[\begin{array}{l}
e^{-t} \cos (t)+\left(2-e^{-t}\right) \sin (t) \\
\left(2+e^{-t}\right) \cos (t)+e^{-t} \sin (t)
\end{array}\right] \quad\left(t \in \mathbf{R}^{+}\right) .
\end{aligned}
$$

In particular, $g(x(t)) \nrightarrow 0(t \rightarrow \infty)$, which would be true if the conclusion of either Theorem 5.2 or Theorem 5.3 were valid (cf. [16, Lemma 1.3]).

6. A theorem on asymptotic stability. Our next goal is to extend [16, Theorem 3.2] to $\mathbf{R}^{n}$. One possible approach is to simply copy [16, Theorem 3.2] (with $\beta=0$ in [16, Condition (H1)]), except that one substitutes ( $\mathrm{H} 2)$ for $g \in C(\mathbf{R} ; \mathbf{R})$. Then the proof in [16] goes through, and one has

TheOREM 6.1. Let (H2), (H4), (H5) and (H6) hold. In addition suppose that $Z(\mu)$ is countable, and that

$$
\hat{\mu}(\omega)=0 \quad(\omega \in Z(\mu)) .
$$

Then every $y \in \Gamma(x)$ is a constant. If moreover $0 \notin Z(\mu)$, then $g(x(t)) \rightarrow 0$ $(t \rightarrow \infty)$.

Theorem 6.1 is true, but not very sharp. In general, a singular matrix does not vanish completely, so (6.1), which, in particular, implies $\hat{\mu}(\omega)+\hat{\mu}^{*}(\omega)=0$ $(\omega \in Z(\mu))$, is rather restrictive. For example, take $n$ linear, real scalar equations of the form $(E)$ :

$$
x_{j}^{\prime}(t)+\int_{[0, t]} x_{j}(t-s) d \mu_{j}(s)=f_{j}(t) \quad\left(j \in \mathbf{N}_{n}\right)
$$

let $x, f$ be the vector-valued functions whose components are $x_{j}, f_{j}$, and let $\mu$ be the diagonal, matrix-valued measure whose diagonal elements are $\mu_{j}$. Then (H4)-(H6) hold, provided $x_{j}, \mu_{j}, f_{j}\left(j \in \mathrm{N}_{n}\right)$ satisfy the corresponding scalar conditions. Assumption (6.1) becomes

(6.2) $\hat{\mu}(\omega)=0 \quad\left(\omega \in Z(\mu)=\left\{\omega \in \mathbf{R} \mid \operatorname{Re} \hat{\mu}_{j}(\omega)=0\right.\right.$ for some $\left.\left.j \in \mathbf{N}_{n}\right\}\right)$.

Thus, if $Z(\mu)$ is countable and (6.2) holds, then Theorem 6.1 applies. However, instead of applying Theorem 6.1 one can apply [16, Theorem 3.2] to the components $x_{j}$ of $x$ separately, and one finds that the conclusion of Theorem 6.1 (ignore its last statement for the moment) remains valid under the weaker assumption $\hat{\mu}_{j}(\omega)=0\left(\omega \in Z\left(\mu_{j}\right)=\left\{\omega \in \mathbf{R} \mid \operatorname{Re} \mu_{j}(\omega)=0\right\}\right)(j \in$ $\left.\mathbf{N}_{n}\right)$. This is equivalent to

$$
\Re(\hat{\mu}(\omega)) \supset \Re\left\{\hat{\mu}(\omega)+\hat{\mu}^{*}(\omega)\right\} \quad(\omega \in \mathbf{R}) .
$$

For a general measure $\mu$ satisfying (H6), condition (6.3) is clearly strictly weaker than (6.1) if $n>2$, but they are equivalent when $n=1$. Hence one 
could expect Theorem 6.1 to remain true if one replaces (6.1) by (6.3). This is indeed the case.

TheOREM 6.2. Let (H2), (H4), (H5) and (H6) hold. In addition suppose that $Z(\mu)$ is countable, and that (6.3) holds. Then every $y \in \Gamma(x)$ is a constant, and the distance from $g(x(t))$ to $\Re\left(\hat{\mu}(0)+\hat{\mu}^{*}(0)\right)$ tends to zero as $t \rightarrow \infty$.

Theorem 6.2 contains Theorem 6.1.

The proofs of [16, Theorem 3.2] and Theorem 6.1 use [16, Lemma 1.12], which in turn depends on a theorem in [3, p. 232], essentially due to Agmon and Mandelbrojt. Here we cannot apply that theorem directly, but have to rewrite its proof to obtain a new version of [16, Lemma 1.12]:

Lemma 6.1. Let $\varphi \in \mathrm{BUC}\left(\mathbf{R} ; \mathbf{C}^{n}\right), \nu, \mu \in \mathrm{BM}\left(\mathbf{R} ; \mathbf{C}^{n, n}\right)$. Moreover, suppose that $\nu * \varphi=0$, and that $\Re(\hat{\mu}(\omega)) \supset \Re(\hat{\nu}(\omega))(\omega \in \mathbf{R})$. Then $\sigma(\mu * \varphi)$ contains no isolated point. If moreover $\sigma(\varphi)$ is countable, then $\mu * \varphi=0$.

Proof of LemMa 6.1. We prove the first claim by showing that if $\omega$ is an isolated point of $\sigma(\mu * \varphi)$, then $\Re(\hat{\mu}(\omega)) \not \mathcal{X}(\hat{\nu}(\omega))$. Let $\omega$ be an isolated point of $\sigma(\mu * \varphi)$. Without loss of generality we can take $\omega=0$ (multiply $\nu, \mu$ and $\varphi$ by $\left.e^{-i \omega t}\right)$. Take some $\varepsilon>0$ such that $[-\varepsilon, \varepsilon] \cap \sigma(\mu * \varphi)=\{0\}$, and choose some $\eta \in \mathcal{S}(\mathbf{R} ; \mathbf{R})$ such that $\hat{\eta}(0)=1, \sigma(\eta) \subset[-\varepsilon, \varepsilon]$. By [15, Lemma 5.2] combined with [16, Lemma 1.11], $\mu * \varphi$ is of the form $\mu * \varphi=\gamma+\psi$, where $\gamma \in \mathbf{C}^{n}, \gamma \neq 0$, and $\psi \in \operatorname{BUC}\left(\mathbf{R} ; \mathbf{C}^{n}\right)$ with $\sigma(\psi) \cap[-\varepsilon, \varepsilon]=\varnothing$. Since $(\eta * \psi)^{\wedge}=\hat{\eta} \hat{\psi}$, and the supports of $\hat{\eta}$ and $\hat{\psi}$ do not intersect, we have $\eta * \psi=0$. This, together with the fact that $\hat{\eta}(0)=1$, gives $\eta * \mu * \varphi=\gamma$. Define $\eta_{k}(t)=k^{-1} \eta(t / k)(t \in \mathbf{R}, k \in \mathbf{N})$. Then $\hat{\eta}_{k}(\omega)=\hat{\eta}(k \omega)(\omega \in \mathbf{R}, k \in$ $\mathbf{N})$, and, in particular, $\hat{\eta}_{k}(0)=1$. Thus also $\eta * \mu * \varphi * \eta_{k}=\gamma(k \in \mathrm{N})$, so clearly

$$
\lim _{k \rightarrow \infty} \eta * \mu * \varphi * \eta_{k}(0)=\gamma \neq 0 .
$$

The set $\left\{\varphi * \eta_{k} \mid(k \in \mathbf{N})\right\}$ is bounded in $L^{\infty}\left(\mathbf{R} ; \mathbf{C}^{n}\right)$, hence weak sequentially relatively compact. Passing to a subsequence (which we to simplify the notations take to be the original one) we can suppose that $\varphi * \eta_{k}$ converges weak ${ }^{*}$ in $L^{\infty}\left(\mathbf{R} ; \mathbf{C}^{n}\right)$ to some function $\xi$. (Here [3, p. 233] contains a misprint; one should define $f_{\varepsilon}=f * \chi_{\varepsilon}$, and not $f_{\varepsilon}=K * f * \chi_{\varepsilon}$.) Since $\sigma\left(\varphi * \eta_{k}\right) \subset$ $[-\varepsilon / k, \varepsilon / k]$ (note that $\left(\varphi * \eta_{k}\right)^{\wedge}=\hat{\varphi} \hat{\eta}_{k}$ and that $\hat{\eta}_{k}$ vanishes outside $-[\varepsilon / k, \varepsilon / k])$ we must have $\sigma(\xi) \subset\{0\}$. Apply [15, Lemma 5.2] to conclude that $\xi$ is a constant. The function $\eta * \mu$ is integrable, and so by (6.4), $\hat{\eta}(0) \hat{\mu}(0) \xi=\gamma \neq 0$. Thus

$$
\xi \notin \Re(\hat{\mu}(0)) \text {. }
$$

On the other hand, $\nu * \varphi=0$, so clearly also $\eta * \nu * \varphi * \eta_{k}=0(k \in \mathrm{N})$. As $\eta * \nu$ is integrable and $\varphi * \eta_{k}$ converges weak ${ }^{*}$ in $L^{\infty}\left(\mathbf{R} ; \mathbf{C}^{n}\right)$ to $\xi$, we have 
$\hat{\eta}(0) \hat{\nu}(0) \xi=0$, i.e. $\xi \in \mathcal{X}(\hat{\nu}(0))$. This together with $(6.5)$ gives $\Re(\hat{\mu}(0)) \not \supset$ $\vartheta(\hat{\nu}(0))$, and completes the proof of the fact that $\sigma(\mu * \varphi)$ contains no isolated point.

The final statement in Lemma 6.1 follows easily. By [16, Lemma 1.9] we have $\sigma(\mu * \varphi) \subset \sigma(\varphi)$, so if $\sigma(\varphi)$ is countable, then $\sigma(\mu * \varphi)$ is closed, countable, and has no isolated point, hence is empty (every nonempty perfect set has more than countably many points). Thus the conclusion of Lemma 6.1 is true.

Proof of Theorem 6.2. As a first step in the proof of Theorem 6.2 we have to sharpen Theorem 5.3. It no longer suffices to know that $\sigma(g \circ y) \subset Z(\mu)$ $(y \in \Gamma(x))$; in addition, we need the fact that

$$
\nu *(g \circ y)=0 \quad(y \in \Gamma(x)),
$$

where $\nu=\mu+\tilde{\mu}$. The proof of (6.6) is essentially the same as in the scalar case, so we do not give it in detail, but only indicate how one should proceed. First use the vector version of [15, Lemma 7.1] to get (4.1) with $\varphi=g \circ x$. Then continue as in $[13, \S 4]$ to conclude that

$$
\sup _{T \in \mathbf{R}^{+}} \int_{\mathbf{R}} \hat{\varphi}_{T}^{*}(\omega) \hat{\nu}(\omega) \hat{\varphi}_{T}(\omega) d \omega<\infty,
$$

where $\varphi_{T}=\chi_{[0, T]} g \circ x$. Since $\hat{\nu} \geqslant 0$ we have $0 \leqslant[\hat{\nu}(\omega)]^{2} \leqslant\|\hat{\nu}(\omega)\| \hat{\nu}(\omega)(\omega \in$ R). Hence also

$$
\sup _{T \in \mathbf{R}^{+}} \int_{\mathbf{R}}\left|\hat{\nu}(\omega) \hat{\varphi}_{T}(\omega)\right|^{2} d \omega<\infty .
$$

Continue as in $[13, \S 4]$ to show that $\nu *(g \circ x)(t) \rightarrow 0(t \rightarrow \infty)$. From this one easily obtains (6.6).

Take some $y \in \Gamma(x)$. By Theorem 5.3 and the countability of $Z(\mu)$ we have $\sigma(g \circ y)$ countable. As, moreover, (6.6) is true we can apply Lemma 6.1 with $\varphi=g \circ y$, and we find that $\mu *(g \circ y)=0$. Hence by (5.1), $y^{\prime}=0$, which proves that every $y \in \Gamma(x)$ is a constant.

We still have to verify the second half of the conclusion of Theorem 6.2. Take some $y \in \Gamma(x)$, necessarily a constant. By (6.6), $\hat{\nu}(0) g(y)=0$, i.e. $g(y) \in \Re(\hat{\nu}(0))=\Re\left(\hat{\mu}(0)+\hat{\mu}^{*}(0)\right)$. If we let $\Pi$ denote the orthogonal projection which has the same nullspace as $\hat{\mu}(0)+\hat{\mu}^{*}(0)$, then we have $\Pi g \circ y=$ $0(y \in \Gamma(x))$. Now use obvious modifications of [16, Lemmas 1.3 and 1.5] to get $\Pi g(x(t)) \rightarrow 0(t \rightarrow \infty)$. This gives the desired conclusion, and completes the proof of Theorem 6.2.

7. Examples on positive definite measures. In $\mathbf{R}^{1}$ it is known that if $d \mu(t)=a(t) d t\left(t \in \mathbf{R}^{+}\right)$, where $a$ is positive, decreasing, and convex, then $\mu$ is positive definite. If, in addition, $a$ is not piecewise linear in a very special way, then $Z(\mu)=\varnothing$. A corresponding result is also true in $\mathbf{R}^{n}$, and even in $\mathbf{C}^{n}$. For simplicity we do as in (H6) and take $a$ integrable on $\mathbf{R}^{+}$: 
Proposition 7.1. Let $d \mu(t)=a(t) d t\left(t \in \mathbf{R}^{+}\right)$, where $a \in L^{1}\left(\mathbf{R}^{+} ; \mathbf{C}^{n, n}\right)$, and suppose that for each $\alpha \in \mathbf{C}^{n}$ the scalar function $\alpha^{*} a \alpha$ is convex. Then $\mu$ is positive definite. Moreover, if there exists some $\alpha \in \mathbf{C}^{n}, \alpha \neq 0$, such that $\alpha^{*} a \alpha=0$, then $Z(\mu)=\mathbf{R}$, otherwise $Z(\mu)=\{\omega \in \mathbf{R}-\{0\} \mid$ there exists $\alpha \in$ $\mathrm{C}^{n}, \alpha \neq 0$, such that $\alpha^{*} a \alpha$ is linear in each interval $(2 \pi(k-1) /|\omega|, 2 \pi k /|\omega|)$ $(k \in \mathbf{N})\}$.

If in Proposition 7.1 one replaces $\mathbf{C}^{n}, \mathbf{C}^{n, n}$ throughout by $\mathbf{R}^{n}, \mathbf{R}^{n, n}$, then one must, in addition, suppose $a=a^{*}$. Proposition 7.1 overlaps [10, Corollary (4.1)].

Proof of Proposition 7.1. Whenever $b \in L^{1}\left(\mathbf{R}^{+} ; \mathbf{R}\right)$ is convex (hence positive, decreasing) one can integrate by parts twice to show that

$$
\operatorname{Re} \hat{b}(\omega)= \begin{cases}\omega^{-2} \int_{(0, \infty)}(1-\cos (\omega t)) d b^{\prime}(t) & (\omega \neq 0), \\ \int_{\mathbf{R}^{+}} b(t) d t & (\omega=0) .\end{cases}
$$

Thus, in particular, $\operatorname{Re} \hat{b} \geqslant 0, \operatorname{Re} \hat{b}(0)=0$ iff $b=0$, and $\operatorname{Re} \hat{b}(\omega)=0$ for some $\omega \neq 0$ iff $b^{\prime}$ is constant in each interval $(2 \pi(k-1) /|\omega|, 2 \pi k /|\omega|)$ $(k \in \mathrm{N})$. This proves Proposition 7.1 in the case $n=1$.

Once the scalar case is known one simply applies Corollary 3.1 to show that the claim $\mu \in \operatorname{PD}\left(\mathbf{R}^{+} ; \mathbf{C}^{n, n}\right)$ is true for any $n \in \mathbf{N}$.

The proof of the claim concerning $Z(\mu)$ can also be based on the preceding scalar argument. First note that

$$
\alpha^{*}\left(\hat{\mu}(\omega)+\hat{\mu}^{*}(\omega)\right) \alpha=2 \operatorname{Re} \alpha^{*} \hat{\mu}(\omega) \alpha=2 \operatorname{Re}\left(\alpha^{*} \mu \alpha\right)^{\wedge}(\omega) \quad(\omega \in \mathbf{R}) .
$$

The matrix $\hat{\mu}(\omega)+\hat{\mu}^{*}(\omega)$ is singular iff there exists some $\alpha \in \mathbf{C}^{n}, \alpha \neq 0$, such that $\alpha^{*}\left(\hat{\mu}(\omega)+\hat{\mu}^{*}(\omega)\right) \alpha=0$. Thus

$$
Z(\mu)=\bigcup_{\substack{\alpha \in C^{n} \\ \alpha \neq 0}}\left\{\omega \in \mathbf{R} \mid \operatorname{Re}\left(\alpha^{*} \mu \alpha\right)^{\wedge}(\omega)=0\right\},
$$

which by the scalar theory is the same set as the one given in Proposition7.1.

There is another important class of kernels in $\mathbf{R}^{1}$ which is known to be positive definite and satisfy the scalar version of (6.1) or (6.3), namely those kernels $\mu$ for which the functions $b(t)=\mu([0, t])\left(t \in \mathbf{R}^{+}\right)$are positive and decreasing on $\mathbf{R}^{+}$. The corresponding matrix-valued kernels are also positive definite. They do not necessarily satisfy (6.1), but they do satisfy (6.3).

Proposition 7.2. Let $\mu \in \mathrm{BM}\left(\mathbf{R}^{+} ; \mathbf{C}^{n, n}\right)$, and suppose that for every $\alpha \in \mathbf{C}^{n}$ the function $\alpha^{*} \mu([0, t]) \alpha\left(t \in \mathbf{R}^{+}\right)$is positive and decreasing. Then $\mu$ is positive definite, and (6.3) holds. Moreover, if $\mu\left(\mathbf{R}^{+}\right)$is nonsingular, then $Z(\mu)=\varnothing$, and otherwise $Z(\mu)=\{0\} \cup\{\omega \in \mathbf{R}-\{0\} \mid$ there exists some $\alpha \in$ 
$\Re\left(\mu\left(\mathbf{R}^{+}\right)\right), \alpha \neq 0$, such that the function $\alpha^{*} \mu([0, t]) \alpha\left(t \in \mathbf{R}^{+}\right)$is constant in each interval $[2 \pi(k-1) /|\omega|, 2 \pi k /|\omega|)(k \in \mathbf{N})\}$.

Proposition 7.2 contains the $\mathbf{R}^{n}$ version of [9, Theorem 2.2] (again define $\left.A(t)=\mu([0, t])\left(t \in \mathbf{R}^{+}\right)\right)$.

Proof of Proposition 7.2. Take some $\alpha \in \mathbf{C}^{n}$ and define $b_{\alpha}(t)=$ $\alpha^{*} \mu([0, t]) \alpha\left(t \in \mathbf{R}^{+}\right)$. Take $\omega \in \mathbf{R}$. Then

$$
\alpha^{*}\left(\hat{\mu}(\omega)+\hat{\mu}^{*}(\omega)\right) \alpha=2 \operatorname{Re}\left(\alpha^{*} \mu \alpha\right)^{\wedge}(\omega)=2 b_{\alpha}(0)+2 \int_{(0, \infty)} \cos (\omega t) d b_{\alpha}(t)
$$

$$
\geqslant 2 b_{\alpha}(0)+2 \int_{(0, \infty)} d b_{\alpha}(t)=2 b_{\alpha}(\infty) \geqslant 0
$$

where we have used the fact that $b_{\alpha}$ is positive and decreasing. Hence by Corollary 3.1, $\mu \in \mathrm{PD}\left(\mathbf{R}^{+} ; \mathbf{C}^{n, n}\right)$.

If $\mu\left(\mathbf{R}^{+}\right)$is nonsingular, then we get equality in (7.1) for no $\alpha \in \mathbf{C}^{n}, \alpha \neq 0$ (because $\left.b_{\alpha}(\infty)>0\right)$, and thus $Z(\mu)=\varnothing$. If, on the other hand, $\mu\left(\mathbf{R}^{+}\right)$is singular, then $\Re\left(\mu\left(\mathbf{R}^{+}\right)\right)$contains some $\alpha \neq 0$. For this $\alpha, b_{\alpha}(\infty)=0$, and one gets equality in (7.1) at least for $\omega=0$. Thus $0 \in Z(\mu)$. In addition, one gets equality for some $\omega \neq 0$ whenever $b_{\alpha}$ is a constant in each interval $[2 \pi(k-1) /|\omega|, 2 \pi k /|\omega|)(k \in \mathbf{N})$.

It only remains to show that (6.3) holds. The measure $\mu$ is selfadjoint (the function $\mu([0, t])\left(t \in \mathbf{R}^{+}\right)$is positive, hence selfadjoint). This implies

$$
\hat{\mu}(\omega)+\hat{\mu}^{*}(\omega)=2 \int_{\mathbf{R}^{+}} \cos (\omega t) d \mu(t) \quad(\omega \in \mathbf{R}) .
$$

Thus, it suffices to show that whenever $\left(\hat{\mu}(\omega)+\hat{\mu}^{*}(\omega)\right) \alpha=0$, then one also has

$$
\int_{\mathbf{R}^{+}} \sin (\omega t) d \mu(t) \alpha=0 .
$$

The case $\omega=0$ is trivial. Take some $\omega \neq 0, \alpha \in \mathbf{C}^{n}$, such that

$$
\left(\hat{\mu}(\omega)+\hat{\mu}^{*}(\omega)\right) \alpha=0 .
$$

Then we have equality in (7.1), so the function $b_{\alpha}$ is constant in each interval $[2 \pi(k-1) /|\omega|, 2 \pi k /|\omega|)(k \in N)$. This means that for every $s \in(0,1), k \in$ $\mathbf{N}$, the matrix $B_{k, s}=\mu((2 \pi(k-1) /|\omega|, 2 \pi(k-1+s) /|\omega|])$ satisfies $\alpha^{*} B_{k, s} \alpha$ $=0$. As, moreover, $B_{k, s} \leqslant 0$, we have $B_{k, s} \alpha=0(s \in(0,1), k \in N)$. Thus the $\mathrm{C}^{n}$-valued measure $\mu \alpha$ vanishes in

$$
\bigcup_{n \in N}(2 \pi(k-1) /|\omega|, 2 \pi k /|\omega|)
$$

But then $\sin (\omega t)$ vanishes a.e. with respect to $\mu \alpha$, so (7.2) holds, and the proof of Proposition 7.2 is complete. 
By Propositions 7.1-7.2 one can apply Theorems 5.1, 5.3 to equations with the two different types of monotone kernels. Of course, if $Z(\mu)=\mathbf{R}$, then the conclusion of Theorem 5.3 becomes trivial. Also Theorem 6.2 applies to equations with monotone kernels of the second type, provided $Z(\mu)$ is countable. The following questions present themselves: Is there anything that can be said about the degenerate case $Z(\mu)=\mathbf{R}$, and is $Z(\mu)$ countable in most cases? The answers to both questions are affirmative:

Proposition 7.3. The kernels in Propositions 7.1-7.2 have either $Z(\mu)=\mathbf{R}$, or $Z(\mu)$ countable. If $Z(\mu)=\mathbf{R}$, then equation $(\mathrm{E})$ can be reduced to an equation in $\mathbf{R}^{n-1}$ (provided $\mu$ is $\mathbf{R}^{n, n}$-valued).

Proof of Proposition 7.3. First consider the kernel in Proposition 7.2, and begin with the case when $\mu(\{0\})$ is singular. Then there exists some $\alpha \in \mathbf{C}^{n}$, $|\alpha|=1$, such that $\mu(\{0\}) \alpha=0$. Note that if $\mu(\{0\}) \in \mathbf{R}^{n, n}$, then one can without loss of generality take $\alpha \in \mathbf{R}^{n}$ instead of $\alpha \in \mathbf{C}^{n}$ (replace $\alpha$ by either $\operatorname{Re} \alpha$ or $\operatorname{Im} \alpha)$. As the function $\alpha^{*} \mu([0, t]) \alpha\left(t \in \mathbf{R}^{+}\right)$is positive and decreasing, we must have $\alpha^{*} \mu([0, t]) \alpha=0\left(t \in \mathbf{R}^{+}\right)$. Thus by Proposition 7.2, $Z(\mu)=\mathbf{R}$.

We claim that in the preceding case one can reduce $(E)$ to an equation in $\mathbf{R}^{n-1}$ (provided $\mu$ is $\mathbf{R}^{n, n}$-valued). Rotating the coordinate system if necessary we can suppose that $\alpha$ is the last base vector in our base. Then $\mu_{n, n}([0, t])=0$ $\left(t \in \mathbf{R}^{+}\right)$, and the positivity of $\mu([0, t])$ yields $\mu_{n j}([0, t])=\mu_{j n}([0, t])=0(j \in$ $\mathbf{N}_{n}, t \in \mathbf{R}^{+}$). Thus (E) becomes

$$
\begin{gathered}
x_{j}^{\prime}(t)+\sum_{k=1}^{n-1} \int_{[0, t]} g_{k}(x(t-s)) d \mu_{j k}(s)=f_{j}(t), \\
x_{j}(0)=x_{j 0} \quad(j=1, \ldots, n-1), \\
x_{n}^{\prime}(t)=f_{n}(t), \quad x_{n}(0)=x_{n 0} .
\end{gathered}
$$

The last equation can be solved trivially, and substituting the value of $x_{n}$ into the first $n-1$ equations we are left with a problem in $\mathbf{R}^{n-1}$.

To complete the proof of Proposition 7.3 for the kernel in Proposition 7.2 it suffices to show that $Z(\mu)$ is countable whenever $\mu(\{0\})$ is nonsingular. Let $T$ be the set $T=\{t \in(0, \infty) \mid \mu(\{t\}) \neq 0\}$ (the set of point masses of $\mu$ ). Then $T$ is countable. We claim that if $\mu(\{0\})$ is nonsingular, then

$$
Z(\mu) \subset\{2 \pi k / t \mid k \in \mathbf{Z}, t \in T\}
$$

Clearly this implies that $Z(\mu)$ is countable.

To prove (7.4) we suppose that $\mu(\{0\})$ is nonsingular, and take $\omega \in Z(\mu)$, $\omega \neq 0$. By Proposition 7.2, there exists some $\alpha \in \mathbf{C}^{n}, \alpha \neq 0$, such that the function $b_{\alpha}(t)=\alpha^{*} \mu([0, t]) \alpha\left(t \in \mathbf{R}^{+}\right)$is constant in each interval

$$
[2 \pi(k-1) /|\omega|, 2 \pi k /|\omega|) \text { and } b_{\alpha}(\infty)=0 \text {. }
$$


On the other hand, $b_{\alpha}(0) \neq 0$, because $\mu(\{0\})$ is nonsingular. Thus there exists $t \in T, k \in \mathrm{N}$, such that $t=2 \pi k /|\omega|$, i.e. $|\omega|=2 \pi k / t$, and the proof is complete.

The kernel in Proposition 7.1 is treated in a very similar way, and we therefore only indicate which changes are necessary in the preceding proof. If one replaces $\mu(\{0\})$ by $\mu\left(\mathbf{R}^{+}\right)$, then the argument for the degenerate case goes through without difficulties. In the case when $\mu\left(\mathbf{R}^{+}\right)$is nonsingular one defines $T=\{t \in(0, \infty) \mid$ the right derivative of $a$ is discontinuous at $t\}$. This set is countable, because $a^{\prime}$ is locally of bounded variation on $(0, \infty)$ (cf. the argument establishing the fact that $\hat{v}$ has order zero in the proof of Theorem 3.2). One completes the proof as above by showing that $Z(\mu) \subset\{ \pm 2 \pi k / t \mid k$ $\in \mathbf{N}, t \in T\}$.

REMARK 7.1. One cannot directly apply Theorems 5.3 and 6.2 to the reduced equation referred to in Proposition 7.3, because the function $g$ in (7.3) depends, in general, not only on $x_{k}(k=1, \ldots, n-1)$, but also on $x_{n}$. One could remove the dependence on $x_{n}$, substituting $x_{n}(\infty)$ for $x_{n}$ and absorbing the error into $f$, but, in general, this destroys (H4). A better way is to work in $\mathbf{R}^{n}$ until one gets (4.1) with $\varphi=g \circ x$, and from then on ignore $x_{n}$ and work in $\mathbf{R}^{n-1}$. One never gets control of the last component $g_{n}$ of $g$, but this does not matter as (7.3) actually is independent of $g_{n}$.

As a final example we study a system of ordinary differential equations

$$
x^{\prime}(t)+A g(x(t))=f(t) \quad\left(t \in \mathbf{R}^{+}\right) ; \quad x(0)=x_{0} .
$$

Proposition 7.4. Let (H2), (H4) hold. In addition, suppose that $A \in \mathbf{R}^{n, n}$, and that $\alpha^{*} A \alpha>0$ for every $\alpha \in \mathbf{R}^{n}, \alpha \neq 0$. Then every bounded solution $x$ of (7.5) satisfies $g(x(t)) \rightarrow 0(t \rightarrow \infty)$.

Proposition 7.4 is undoubtedly known, and here it only serves as an illustration of Theorem 6.1. Note, in particular, that we do not require any symmetry of $A$. Proposition 7.4 follows from Theorem 6.1 , because if one defines $d \mu(t)=A \delta_{0}$, where $\delta_{0}$ is the scalar Dirac measure at zero, then (7.5) is transformed into an equation of the form (E) with $\hat{\mu}(\omega)+\hat{\mu}^{*}(\omega)=A+A^{*}$ $(\alpha \in \mathbf{R})$ and

$$
\alpha^{*}\left(A+A^{*}\right) \alpha=2(\operatorname{Re} \alpha)^{*} A(\operatorname{Re} \alpha)+2(\operatorname{Im} \alpha)^{*} A(\operatorname{Im} \alpha)>0
$$

for every $\alpha \in \mathbf{C}^{n}, \alpha \neq 0$.

\section{REFERENCES}

1. V. Barbu, Integro-differential equations in Hilbert spaces, An. Ști. Univ. "Al. I. Cuza" Iaşi Sect. I a Mat. 19 (1973), 365-383.

2. , Nonlinear Volterra equations in a Hilbert space, SIAM J. Math. Anal. 6 (1975), $728-741$.

3. W. F. Donoghue, Jr., Distributions and Fourier transforms, Academic Press, New York, 1969.

4. J. K. Hale, Sufficient conditions for stability and instability of autonomous functional-differen- 
tial equations, J. Differential Equations 1 (1965), 452-482. MR 32 \# 1414.

5. J. J. Levin, On some geometric structures for integrodifferential equations, Advances in Math. (to appear).

6. J. J. Levin and D. F. Shea, On the asymptotic behavior of the bounded solutions of some integral equations. I, II, III, J. Math. Anal. Appl. 37 (1972), 42-82, 288-326, 537-575. MR 46 \#5971.

7. S.-O. Londen, On an integral equation in a Hilbert space, SIAM J. Math. Anal. (to appear).

8. __ An existence result on a Volterra equation in a Banach space, Trans. Amer. Math. Soc. (to appear).

9. R. C. MacCamy, Nonlinear Volterra equations on a Hilbert space, J. Differential Equations 16 (1974), 373-393.

10. R. C. MacCamy and J. S. W. Wong, Stability theorems for some functional equations, Trans. Amer. Math. Soc. 164 (1972), 1-37. MR 45 \#2432.

11. J. A. Nohel and D. F. Shea, Frequency domain methods for Volterra equations, Advances in Math. (to appear).

12. L. Schwartz, Théorie des distributions, new ed., Hermann, Paris, 1966. MR 35 \# 730.

13. O. J. Staffans, Nonlinear Volterra integral equations with positive definite kernels, Proc. Amer. Math. Soc. 51 (1975), 103-108.

14. , Positive definite measures with applications to a Volterra equation, Trans. Amer. Math. Soc. 218 (1976), 219-237.

15. , Tauberian theorems for a positive definite form, with applications to a Volterra equation, Trans. Amer. Math. Soc. 218 (1976), 239-259.

16. , On the asymptotic spectra of the bounded solutions of a nonlinear Volterra equation, J. Differential Equations (to appear).

17. , An inequality for positive definite Volterra kernels, Proc. Amer. Math. Soc. 59 (1976), 205-210.

18. D. G. Weis, Note on a Volterra integro-differential equation system, Proc. Amer. Math. Soc. 45 (1974), 214-216. MR 50 \# 14140.

Institute of Mathematics, Helsinki University of Technology, SF-02150 Espoo 15, FinLAND 